\title{
Beneficios del turismo científico en un paisaje forestal mega-diverso: Caso comunidad Kichwa San José de Payamino, Amazonía Ecuatoriana
}

\section{Benefits of scientific tourism in a mega-diverse forest landscape: Case of the Kichwa community in San José De Payamino, Ecuadorian Amazon}

\author{
Gabriela Izurieta $^{1 *}$, Alexandra Torres ${ }^{2,3}$, Yudel García ${ }^{2,3}$, Julio C. Vargas ${ }^{2}$, Sandra Soria ${ }^{2}$, Javier Patiño ${ }^{4}$ y Bolier Torres ${ }^{2,3}$ \\ ${ }^{1}$ Graduada Maestría en Turismo, Universidad Estatal Amazónica, Pastaza 160101, Ecuador \\ ${ }^{2}$ Universidad Estatal Amazónica, UEA, Pastaza 160101, Ecuador \\ ${ }^{3}$ Programa de Economía de Recursos Naturales y Desarrollo Empresarial, UEA, Ecuador \\ ${ }^{4}$ Administrador Estación Biológica Timburi Cocha, UEA, Orellana, Ecuador \\ *Correspondencia: gaby.izurieta19@gmail.com
}

Rec.: 09.04.2019. Acept.: 18.01.2020. Publicado el 30 de junio de 2020

\section{Resumen}

$\mathrm{E}^{1}$ presente documento analiza los beneficios del turismo científico (TCi) a nivel comunitario, desde los puntos de vista, social, económico, ambiental y cultural, realizado por la Estación Biológica Timburi Cocha (EBTC) en la comunidad Kichwa San José de Payamino. La EBTC se localiza en la zona de amortiguamiento del Parque Nacional Sumaco Napo Galeras (PN-SNG) en la Reserva de Biosfera Sumaco (RBS), formando parte del hotspot Biodiversidad (Andino-Amazónico). Se utilizó la metodología de observación científica, encuestas a nivel de hogares y entrevistas a nivel de los líderes comunitarios y dirigentes de entidades académicas involucradas con la EBTC. Los resultados muestran que las actividades del TCi realizado en la EBTC ha contribuido a la comunidad San José de Payamino en un promedio anual que van desde los USD 3474 a 5000 en los últimos ocho años, además de otros beneficios económicos, sociales, ambientales y culturales como producto del involucramiento de los miembros de la comunidad en las diferentes actividades que realizan la EBTC. Los "Líderes Comunitarios" revelaron ponderaciones de "alta" a los temas relacionados con: a) respeto al conocimiento ancestral; b) respeto y conservación a los RRNN; c) empoderamiento o respeto a la identidad cultural; d) respeto a la lengua kichwa y; d) participación de miembros de la comunidad en las actividades realizadas en la EBTC, mientras que; e) aprendizaje mutuo obtuvo una ponderación de "media". Finalmente, el documento presenta recomendaciones para mejorar los beneficios de las actividades del TCi realizados en la EBTC, así como también para contribuir con el desarrollo sostenible y la conservación de los recursos naturales de la comunidad.

Palabras claves: Reserva de Biosfera, hotspot de biodiversidad, conservación, desarrollo sostenible.
$T$ his document analyses the benefits of scientific tourism (ScT) at a community level from a social, economic, environmental and cultural points of view. The research was carried out at the Timburi Cocha Biological Station (TCBS) within the Kichwa community of San José de Payamino, located in the buffer zone of the Sumaco Napo Galeras National Park (SNG-NP) in the Sumaco Biosphere Reserve (SBR) and is part of one of the world's Andean-Amazonian biodiversity hotspots. We employed the methodology of scientific observation, household survey and interviewing community leaders and leaders of the two main academic entities involved with the TCBS. The results show that the activities of ScT has contributed to the San José de Payamino community with an annual average ranging from 3474 to 5000 USD in the last eight years. In addition to other economic, social, environmental and cultural benefits as a result of the involvement of community members in the different activities carried out by the EBTC. The "Community Leaders" revealed "high" weights on issues related to: a) respect for ancestral knowledge; b) respect and conservation of NRNs; c) empowerment or respect for cultural identity; d) respect for the Kichwa language and; d) participation of community members in the activities carried out in the EBTC, while; e) mutual learning obtained a "medium" weighting. Finally, the document presents recommendations to improve the benefits of the TCi activities carried out in the EBTC, as well as to contribute to the sustainable development and conservation of the community's natural resources.

Keywords: Biosphere Reserve, biodiversity hotspot, conservation, sustainable development. 


\section{Introducción}

E n la literatura especializada, el turismo científico aparece a mediados de la década de los 90 con el interés de fomentar la cultura científica, lucha contra la pobreza, exclusión social vinculado con la conservación y respeto ambiental (García, Martínez, 2017). El turismo científico involucra a la comunidad y sus visitantes en las actividades de exploraciones e investigaciones científicas (García, Martínez, 2017). Tiene la finalidad de consolidar los productos y la oferta del turismo científico, certificando la calidad científica en los proyectos realizados (Bourlon, Mao y Quezada, 2013) son aquellas actividades con objetivos de investigación científica que incluye la participación de turistas, estudiantes y/o especialistas que aportan de las diferentes formas de desarrollo de la investigación (Bourlon, Mao y Quezada, 2013), añadiendo una experiencia única y enriquecedora para el turismo.

El turismo científico ayuda a ampliar conocimientos de investigación, motivado por el turismo de aventura, ecoturismo que es añadido por una perspectiva de aprendizaje para quienes practican la investigación científica (García, Martínez, 2017). El Turismo Científico lo conforman estudiantes, profesores e investigadores que visitan diversos lugares con características naturales para realizar los análisis investigativos científicos (Quesada, R. 2010).

A nivel del desarrollo comunitario, se puede mencionar que el TCi puede insertarse como una forma del turismo comunitario. El Turismo Comunitario se establece como estrategia de desarrollo y objeto de investigación científica al demostrar la esencia de su vivencia diaria, cultura, cosmovisión y autenticidad de vida diferente al mundo globalizado (Ródas, Sanmartín \& Ullauri, 2015). Se trata de implementar un turismo que logra equilibrar lo medio ambiental y cultural con el protagonismo de las comunidades que son objetos de desarrollo antes que sujetos del mismo y Ecuador es una muestra de ello (Ruiz \& Solis, 2016).

Dentro de América Latina, Ecuador se considera como el país pionero, desde el aspecto cualitativo más que cuantitativo de Turismo Comunitario, ha beneficiado de varias maneras a las comunidades fortaleciendo el empoderamiento, autoestima, creando políticas democráticas, equitativas y mejorando la calidad de vida (Amstrong, 2012). En lo económico contribuye a la reducción de la pobreza con la finalidad de erradicarla, generando empleo en sus familias y promoviendo el fortalecimiento de la gobernanza local (Bursztyn, Bartholo y Gruber, 2009).

En los últimos años, las universidades nacionales e internacionales se están convirtiendo en un actor importante para fomentar el TCi a nivel de comunidades con alto patrimonio natural y cultural, es así que han empezado a ofertar programas de capacitación en cursos de verano e investigación in situ, con los objetivos de mejorar los programas de capacitación en carreras de ciencias de la vida y de la tierra, así como también contribuir al avance de la ciencia en estos temas, para lo cual, la presencia de estaciones biológicas y/o científicas involucrando a comunidades locales se constituyen en un factor clave (Revelo, 2017). En Ecuador, se han desarrollado varias iniciativas de estaciones científicas ubicadas en la Amazonía, y al menos 10 de éstas son administradas o vinculadas a universidades (Silva, 2018). Sus actividades e investigaciones tienen como objetivo no solo la conservación de especies, sino el desarrollo de nuevas tecnologías y conocimientos (López, 2013).

Entre las principales universidades de Ecuador que realizan cursos de capacitación e investigaciones en estaciones científicas involucrando áreas del Sistema Nacional de Áreas Protegidas (SNAP), Reservas de Biosferas, bosques protectores o zoológicos en la región Amazónica, ya sea a través de convenios con entidades públicas o privadas, nacionales o internacionales tenemos: Universidad Internacional SEK (Reserva Biológica Limoncocha), Universidad San Francisco de Quito y Universidad Católica del Ecuador (Reserva de Biosfera Yasuní y Parque Nacional Yasuní), Universidad Central del Ecuador (Bosque Protector CEPLOA), Universidad Tecnológica Equinoccial (Estación Biológica Pindo Mirador), Escuela Superior Politécnica de Chimborazo (Centro Zoológico Fátima, Pastaza), Universidad Politécnica Salesiana (Cordillera Kutukú), Universidad Regional Amazónica IKIAM (Reserva Biológica Colonso-Chalupas), Universidad Nacional de Loja (Estación experimental El Padmi) (Silva, 2018), y la Universidad Estatal Amazónica (Estación Biológica Timburi Cocha en la Reserva de Biosfera Sumaco, Estación Biológica Nangaritza y Centro de Investigación, Posgrado y Conservación Amazónica, CIPCA).

En este contexto y tomando como caso de estudio la comunidad Kichwa San José de Payamino, el presente artículo tiene como objetivos: a) describir las principales características socio demográficas de la comunidad San José de Payamino; b) analizar los beneficios económicos asociados a las actividades realizadas en la EBTC en la comunidad de Payamino; c) describir los principales atractivos turísticos mayormente visitados en la comunidad; d) analizar las percepciones del sector académico y el sector comunitario sobre las actividades de TCi realizados en la EBTC. 


\section{Materiales y métodos}

$\mathrm{S}_{\mathrm{a}}^{\mathrm{e}}$ e realizaron encuestas a 62 hogares de la comunidad, a partir de las cuales se analizó las condiciones de vida rurales, la dependencia de los recursos naturales y la percepción de los habitantes de esta comunidad en torno a las actividades del turismo científico realizado desde hace 10 años a través de la Estación Biológica Timburi Cocha (EBTC).

La investigación se realizó en la comunidad Kichwa San José de Payamino ubicado en el cantón Loreto, provincia de Orellana, a $304 \mathrm{msnm}$ (Figura 1). San José de Payamino se encuentra dentro de la zona de amortiguamiento del Parque Nacional Sumaco Napo Galeras (PNSNG) y de la Reserva de Biosfera Sumaco (RBS). Esta zona también forma parte del hotspot Andino-Amazónico, el cual es considerado una de las regiones biológicamente más ricas del planeta, famoso por su variedad de especies de flora y fauna. Actualmente, esta área constituye, en su mayoría, un mosaico de bosques naturales y pequeños predios con sistemas productivos de subsistencia.

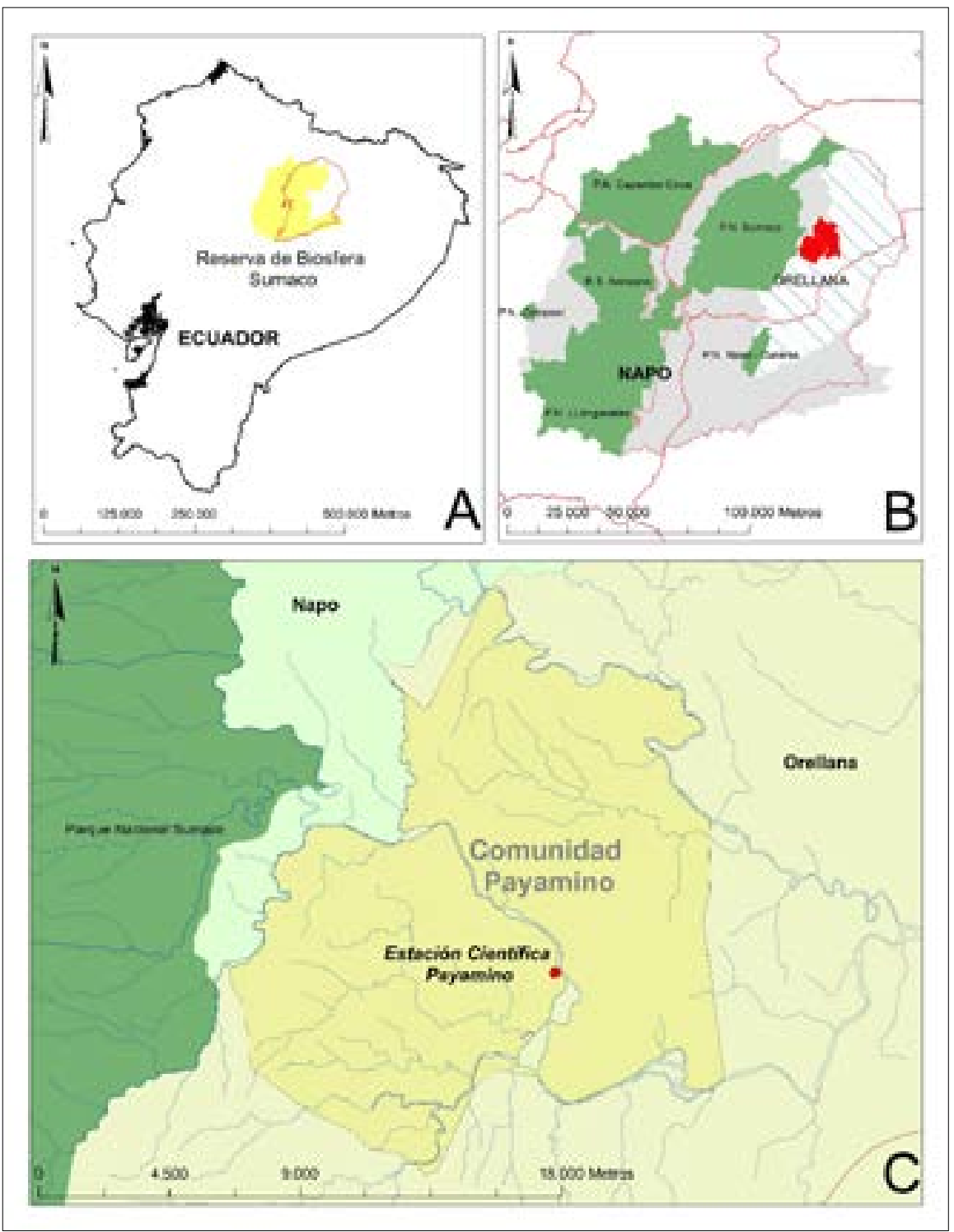

Figura 1: Ubicación geográfica del área de estudio: A) Ecuador y la Reserva de Biosfera Sumaco (RBS); B y C), RBS y áreas protegidas y localización de la Comunidad Kichwa San José de Payamino, cantón Loreto, Orellana, Ecuador, 2018 
Los principales usos del suelo en San José de Payamino son los bosques nativos que cubren un $80 \%$ de área total de la comunidad Kichwa San José de Payamino, el río Payamino que atraviesa la comunidad y que constituye un importante recurso para la pesca artesanal de sus habitantes para su consumo diario y cultivos de cacao, café, naranjilla, maíz, yuca y plátano y palma de chonta que normalmente la cultivan dentro del sistema agrícola tradicional denominado chakra (Figura 2).

Análisis de percepción de los actores claves de la EBTC

La gestión de la Estación Biológica Timburi Cocha se articula en una pequeña red de actores conformada por organizaciones científicas y representantes comunitarios que están relacionados. Para analizar las percepciones de estos actores claves, se realizaron entrevistas que miden la percepción de los actores claves a las actividades que realiza la Estación Biológica Timburi Cocha (Figura 3)

\section{Resultados y discusión}

\section{Características socio demográficas de la comunidad}

De los resultados de la encuesta se determinaron las principales características demográficas de la comunidad Kichwa San José de Payamino (Cuadro 1). El total de la población al momento de realizada la encuesta era de 361 personas, siendo el promedio del tamaño del hogar de 6\%; mientras que el tamaño de hogar de adulto equivalente (AE) es de $4.3 \%$, lo cual es similar a lo reportado por otros estudios en la Amazonía

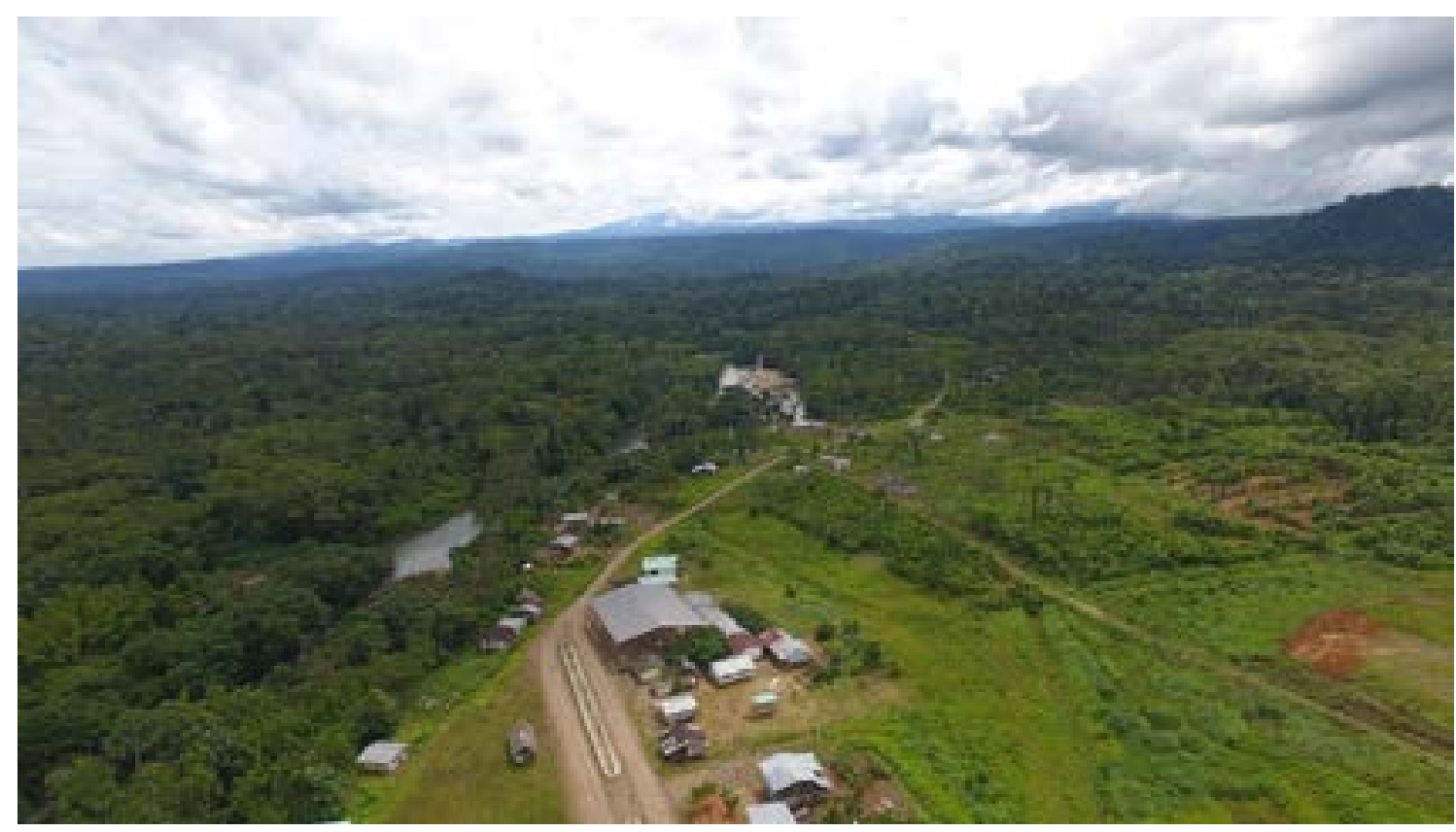

Figura 2: Comunidad Kichwa San José de Payamino cantón Loreto, Orellana, Ecuador, 2018

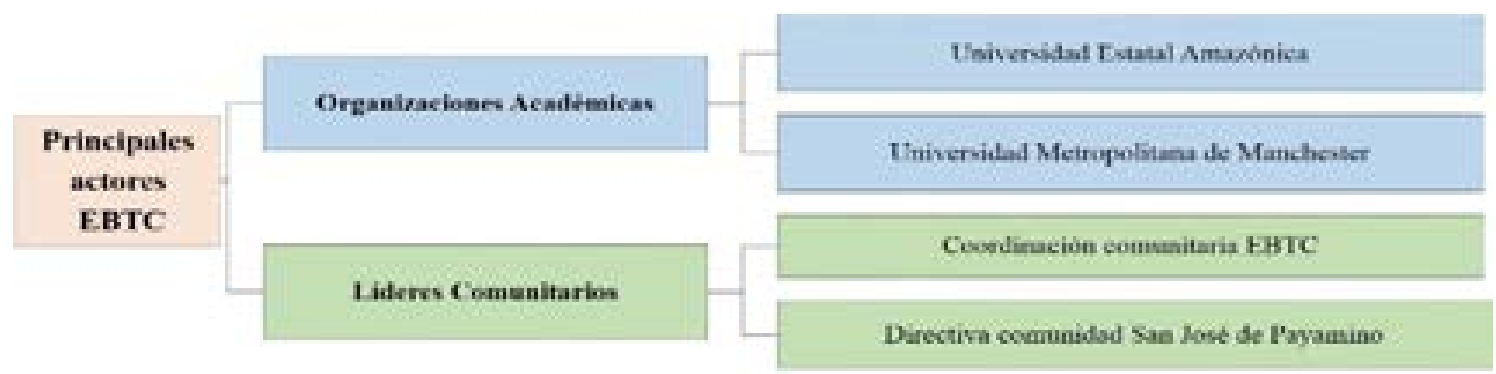

Figura 3: Principales actores para la gestión de la EBTC en la Comunidad San José de Payamino, cantón Loreto, Orellana, Ecuador, 2018 
Ecuatoriana para poblaciones Kichwas (Vasco et al., 2017; Vasco et al., 2015) el promedio de edad del jefe de hogar es de 43 años con un mínimo de 20 y máximo de 80 años, haciendo de esto una población relativamente joven.

En lo referente al capital humano, el nivel de educación del jefe de hogar es de 7 años (primaria), lo cual limita encontrar empleos asalariados (Cuadro 2). La mayoría de los habitantes (84\%) nacieron en la comunidad, esto se refleja también en el hecho de que la mayoría de la población (82\%) confía en sus vecinos y el $69 \%$ hayan recibido ayuda en ocasiones de emergencia o por necesidad por parte de sus vecinos o de la comunidad. Estos porcentajes son altos si se considera otros resultados en la zona de estudio como lo reportado por (Torres et al., 2018) en un estudio realizado en toda la RBS, donde en promedio el $65 \%$ de la población tenía confianza en sus vecinos. En general los habitantes mencionaron que la vida en la comunidad es estable y placentera (Cuadro 1).

\section{Beneficios económicos del TCi hacia la comunidad}

De acuerdo a los datos obtenidos se puede apreciar que las universidades Metropolitana de Manchester
(Inglaterra), Glasgow (Escocia), Alma College, Biodiversity Group (USA) y la Universidad Estatal Amazónica (Ecuador) visitan la Estación Biológica, para realizar cursos de verano con distintas actividades relacionadas con estudios de: plantas medicinales, mastofauna, microfauna, herpetofauna, avifauna, geografía humana, uso de tecnología para monitorear biodiversidad, etc. Así como también diferentes prácticas de campo relacionadas con sistemas de agricultura tradicional, soberanía alimentaria y saberes ancestrales. En todas estas actividades, anualmente visitan la EBTC entre 50 a 150 estudiantes y académicos, permaneciendo en la estación entre 20 a 150 días al año.

Para determinar los beneficios económicos de las actividades del TCi en la EBTC, se realizó la revisión de los registros de ingresos y pagos realizados a la comunidad San José de Payamino por las visitas de estudiantes e investigadores de las universidades antes mencionadas desde el 2011 al 2018.

Uno de los ingresos más importante que genera las distintas actividades consideradas en este documento como TCi en la EBTC, es el pago por el ingreso a la comunidad y sus sitios determinados para realizar ensayos de investigaciones y actividades de exploración.

Cuadro 1. Características demográficas de la comunidad San José de Payamino, Loreto, Orellana, Ecuador, 2018

\begin{tabular}{lcccc}
\hline \multicolumn{1}{c}{ Variables } & Promedio & DS & Max & Min \\
\cline { 2 - 5 } & & & & \\
\hline Variables demográficas & & & & \\
Tamaño del hogar & 5.82 & 2.90 & 14 & 1 \\
Tamaño del hogar (AE) & 4.37 & 1.97 & 10 & 1 \\
Edad / jefe de hogar (años) & 43.37 & 13.47 & 80 & 20 \\
Años educación / jefe hogar (años) & 6.50 & 3.53 & 13 & - \\
Nació en comunidad (\%) & 83.9 & 0.37 & - & - \\
Capital social & & & & - \\
Confía en vecinos (\%) & & & - & - \\
Si & 82.3 & - & - & \\
Un poco & 16.1 & - & - & - \\
No & 1.6 & - & - \\
Recibe ayuda (\%) & & & - \\
Si & 69.4 & - & - \\
Un poco & 27.4 & - & - \\
No & 3.2 & & - & \\
\hline
\end{tabular}


Este pago se encuentra reglamentado y acordado entre la Comunidad y la Administración de la EBTC, iniciando con un pago diario US \$ 3 desde el año 2011 al 2012; US \$ 5 desde el 2013 al 2015, mientras que desde el 2016 este pago se incrementó a US \$ 7 por persona/ día. Este pago por el ingreso a la EBTC contribuye a la comunidad San José de Payamino con un promedio anual que va desde los US \$ 3474 a 5000 en estos años mencionados (Cuadro 2). De esta manera, estos ingresos adicionales sirven a los miembros de la comunidad para cubrir necesidades y disminuir la tala de árboles.

Además del pago por ingreso a la Comunidad donde se encuentra la EBTC, los miembros de la comunidad también se benefician de ingresos económicos derivados de distintas actividades propias del TCi. Dependiendo de cada actividad, cada persona involucrada recibe un ingreso económico que varía dependiendo del servicio prestado, por ejemplo, la mano de obra en general se paga US \$ 17 el día por persona, excepto para el servicio de cocina, que se paga US $\$ 25$ al día, y al ayudante de cocina US \$20 (Cuadro 3).

Sin embargo, la movilización en canoa es otro rubro importante para los miembros de la comunidad, el cual varía dependiendo el lugar que se va a visitar, por ejemplo, el costo para el traslado de visitantes desde la EBTC hasta el saladero para avistamiento de loros es de US \$ 100 , mientras que para llegar hasta las cavernas y laguna del río Cachiyacu es de US \$ 135 y, hasta la laguna Timburi Cocha y otras cascadas en el mismo sector tiene un valor de US \$200, incluyendo al ayudante del canoero.

Cuadro 2. Promedio de ingresos anuales derivados de las actividades del TCi en la EBTC en beneficio de la comunidad San José de Payamino, cantón Loreto, Orellana, Ecuador, 2018.

\begin{tabular}{ccccc}
\hline Año & Estudiantes & Días & $\begin{array}{c}\text { Pago } \\
\text { diario } \\
\text { US \$ }\end{array}$ & $\begin{array}{c}\text { Total } \\
\text { ingresos } \\
\text { US \$ }\end{array}$ \\
\hline 2011 & 66 & 59 & 3 & 3474 \\
2012 & 96 & 84 & 3 & 3931 \\
2013 & 94 & 147 & 5 & 5000 \\
2014 & 58 & 39 & 5 & 3740 \\
2015 & 52 & 28 & 5 & 3530 \\
2016 & 59 & 37 & 7 & 4879 \\
2017 & 49 & 24 & 7 & 3507 \\
2018 & 50 & 46 & 7 & 3934 \\
\hline
\end{tabular}

Destinos de los ingresos comunitarios por la EBTC

Los beneficios económicos derivados por el $\mathrm{TCi}$ realizados en la EBTC son administrados por la dirigencia de la Comunidad San José de Payamino, $\mathrm{y}$ generalmente las decisiones las toman en las Asambleas de la comunidad. De esta manera, en esta investigación se reportan al menos cuatro principales tipos de inversiones que la comunidad ha realizado en los últimos diez años con los recursos obtenidos de la EBTC: a) realizar trámites legales y administrativos en las ciudades de Loreto y Coca; b) ayudar a los hogares de la comunidad en la implementación de cultivos agrícolas; c) inversiones para realizar mingas dentro de la comunidad; d) emergencias médicas o enfermedades a miembros de la comunidad.

Como caso excepcional, en los años 2014 y 2015 se utilizó el dinero obtenido por las actividades del TCi para comprar los útiles escolares para niños miembros de la comunidad que estaban en la escuela. En resumen, desde el 2011 hasta 2018 en promedio la comunidad ha recibido 31995 US \$/anual.

Principales atractivos turísticos y actividades de TCi en la comunidad Kichwa San José de Payamino

Los principales atractivos turísticos de la comunidad Kichwa San José de Payamino son parte del patrimonio natural que poseen, son aprovechados de manera sostenible principalmente por los estudiantes e investigadores que visitan la EBTC, y se han convertido también en un ingreso económico para la población de la comunidad. Al momento no existe un inventario turístico con las recomendaciones del Ministerio de

Cuadro 3. Actividades y valores pagados en USD/ diario a miembros de la comunidad relacionadas con la EBTC. San José de Payamino, Loreto, Orellana, Ecuador, 2018

\begin{tabular}{cl}
\hline $\begin{array}{c}\text { Valor pagado en } \\
\text { USD/diario }\end{array}$ & \multicolumn{1}{c}{ Actividades } \\
\hline 17 & Guía de turismo \\
17 & Canoeros \\
17 & Ayudante de canoero \\
25 & Cocineras expertas \\
20 & Ayudante de cocina \\
17 & Limpieza \\
17 & Construcción \\
17 & Mantenimiento \\
17 & Guía de campo \\
- & Secretaria \\
17 & Investigaciones con voluntarios \\
\hline
\end{tabular}


Turismo, pero la población local realiza actividades haciendo un pequeño recorrido según como desean los turistas que los visitan. En la Figura 4 se muestra los
10 principales atractivos turísticos que son mayormente visitados por los visitantes de la EBTC en Payamino.
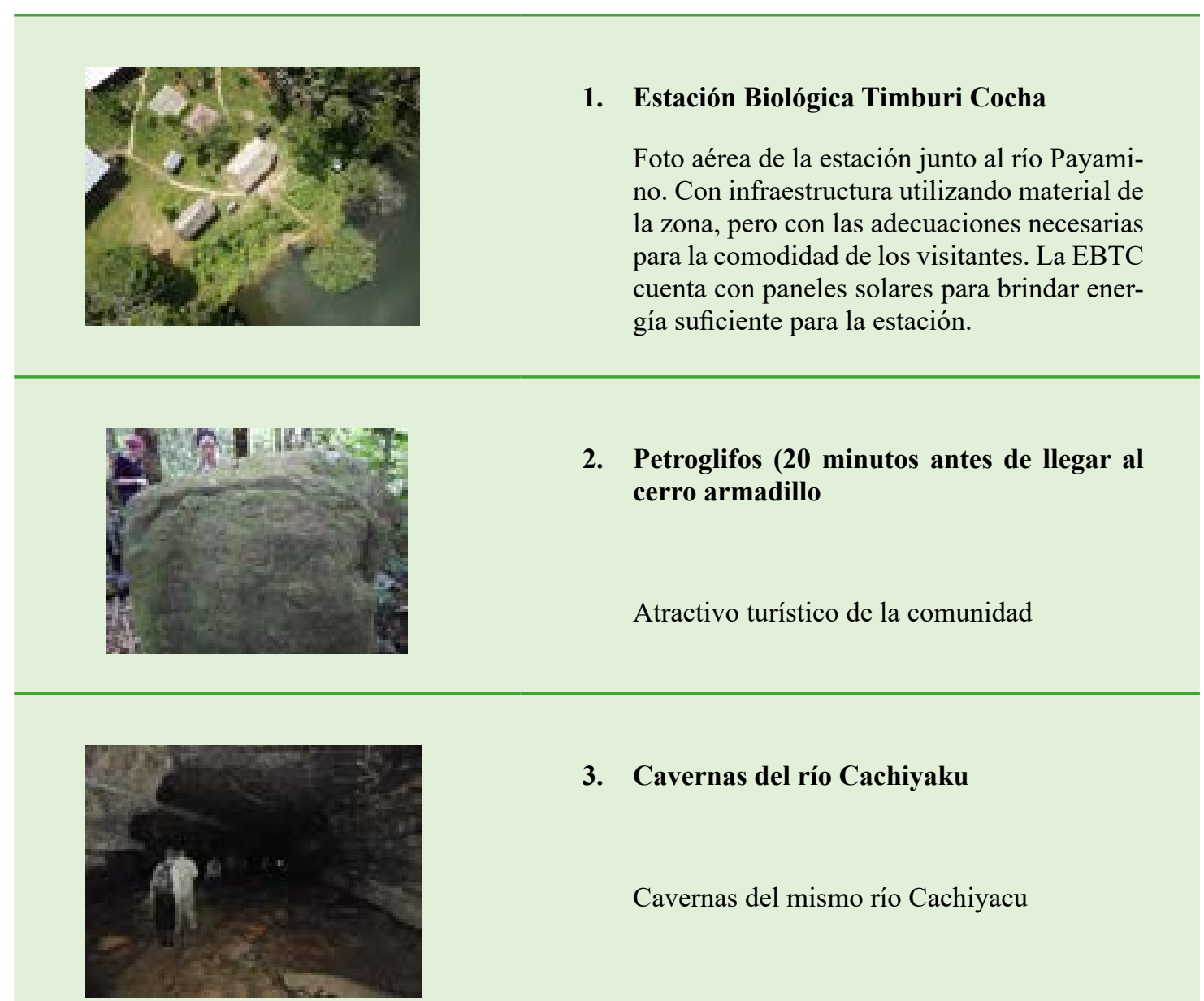

\section{Cavernas del río Cachiyaku}

Cavernas del mismo río Cachiyacu

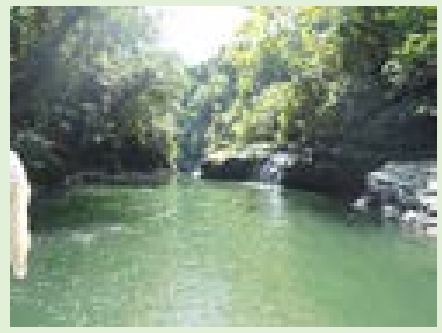

\section{Laguna del rio Cachiyaku}

Atractivo turístico a visitar Cachiyacu a 4 horas en canoa

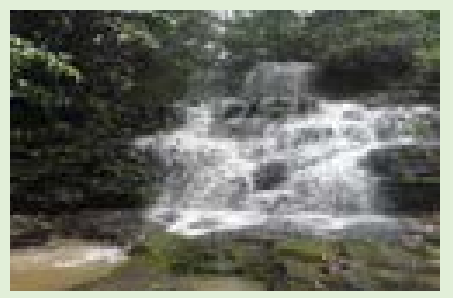

\section{Cascada rio Payamino}

Considerado también un atractivo turístico las cascadas del río Payamino

\section{Figura 4. A Continuación...}




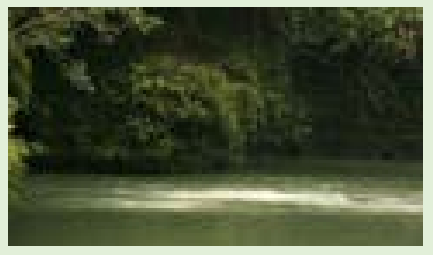

\section{Laguna Timburi Cocha}

Su nombre Timburi Cocha original en kichwa y traducido al español significa laguna burbujeante es el atractivo principal de la comunidad ubicada a 6 horas en canoa

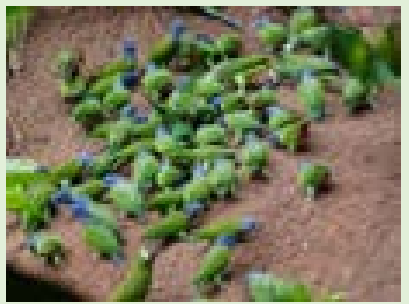

2. Sitio de investigaciones de aves cerca de la estación

La Universidad de Glasgow desde el año 2000 ha contribuido con el inventario de especies de aves de la Región Sumaco, 339 aves de 64 especies fueron inventariadas 10 de las cuales eran nuevas especies en Payamino encontradas en el 2012.

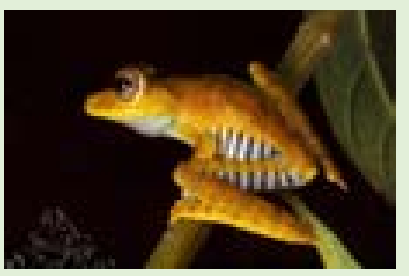

3. Sitios de estudios de herpetofauna (ranas) en bosques primario, secundario y chakras

Se encuentran alrededor de 129 individuos de 22 especies diferentes, las especies más abundantes en 2012 fue Rhinella margaritifer con 34 individuos capturados para sus análisis.

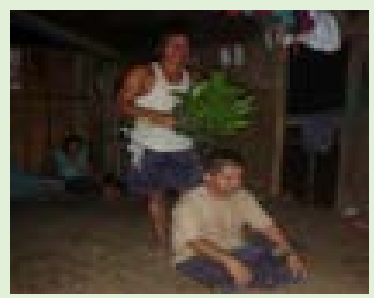

4. Shamanismo

Parte de la tradición de la cultura kichwa, realizado por el shaman de la comunidad

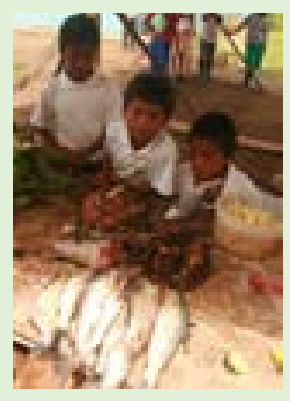

5. Mikuna tradicional

Comida tradicional de la comunidad, maitos de pescado, maito de pollo, chicha de yuca, chicha de chonta, chontacuros y productos de la zona.

Figura 4: Principales atractivos turísticos donde se ejecutan actividades de TCi en la comunidad San José de Payamino. Fotos: Registros de la EBTC, Loreto, Orellana, Ecuador, 2018 
Percepción sobre Turismo de Investigación Científica

El sector académico que participan de alguna manera en la EBTC percibe en un nivel de “alto" (Figura 5). Esto se debe a que las organizaciones científicas que forman parte de la EBTC, distinguen que las acciones de TCi contribuyen no solo a la gestión comunitaria en el buen manejo de los recursos naturales, sino también en la divulgación científica de los resultados de las investigaciones realizadas. La percepción de este sector es que la divulgación científica ejerce un impacto positivo en todos los demás actores, mismos que al conocer las potencialidades de estos bosques en materia de flora y fauna y la importancia de los saberes ancestrales, contribuyen con información primaria para la toma de decisiones en beneficio de los recursos naturales y desarrollo sostenible de la población local. Para este sector, el valor más alto de todas las formas del TCi se muestra en la conservación de los recursos naturales

Estos resultados también se atribuyen al nivel de especialización que tienen los visitantes, especialmente porque la mayoría viene a tomar cursos de verano correspondiente a las carreras de ciencias biológicas o afines, provenientes principalmente de las universidades de Manchester, Shefield (Inglaterra); Glasgow (Escocia); Alma College y Biodiversity Group (USA) y Universidad Estatal Amazónica (Ecuador).

Sin embargo, en este estudio, la percepción de los "Líderes Comunitarios" usando las cuatro formas de TCi: exploración y aventura, cultural científico, eco-voluntariado, investigación científica (Bourlon \& Mao, 2011), reveló ponderaciones de alta y media (Figura 5). Las categorías que registraron valores altos fueron: respeto del conocimiento ancestral, respeto y conservación a los RRNN, empoderamiento o respeto a la identidad cultural, respeto a la lengua kichwa y la participación de miembros de la comunidad en las actividades realizadas en la EBTC. Mientras que el aprendizaje mutuo reveló ponderación media (Figura 5).

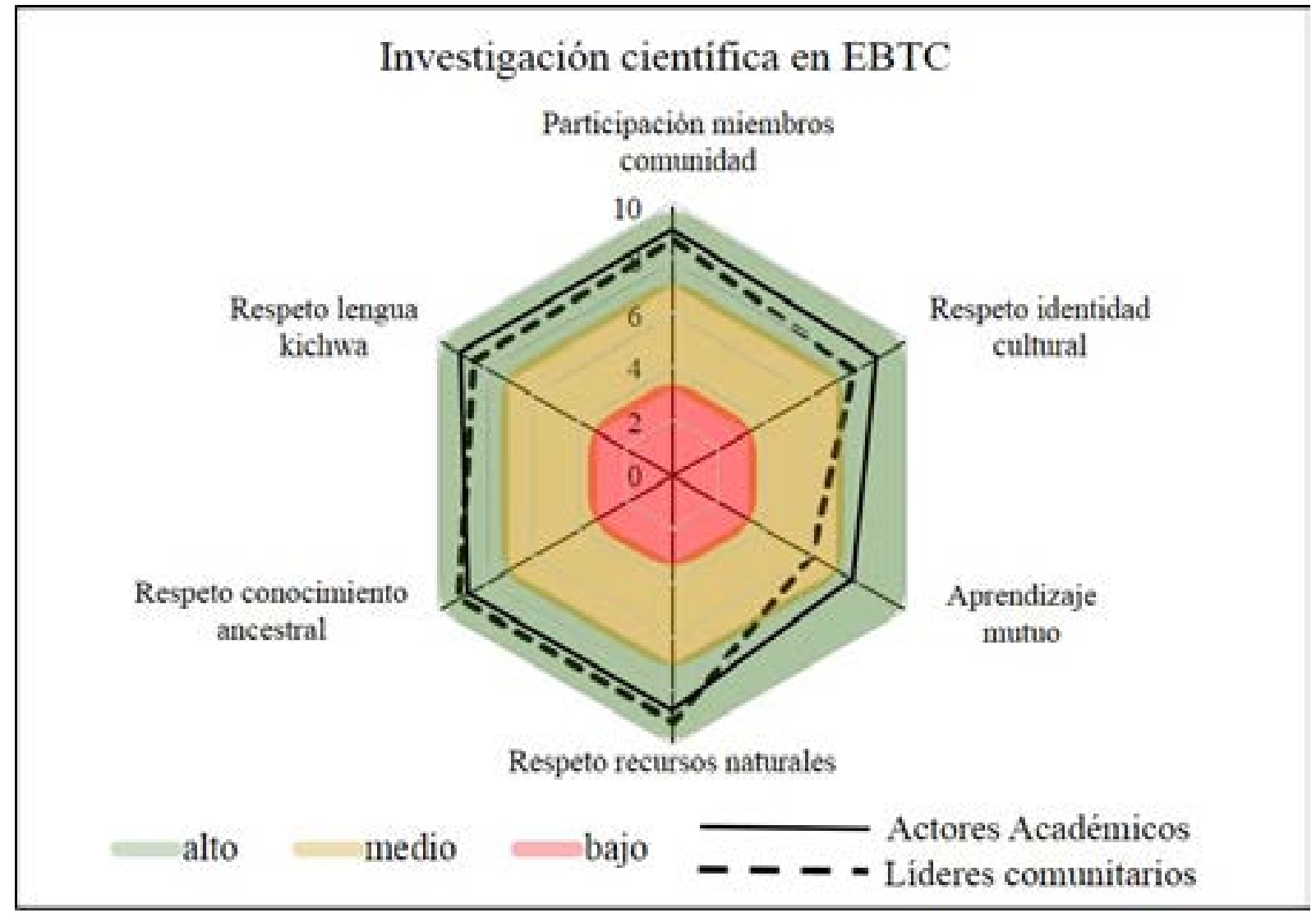

Figura 5: Percepción sobre el turismo científico y el respeto a los elementos de la cosmovisión indígena por parte de los actores académicos y líderes comunitarios de la Comunidad San José de Payamino, cantón Loreto, Orellana, Ecuador, 2018. 


\section{Conclusiones y recomendaciones}

Tos resultados demográficos indican que es una Lpoblación relativamente joven y que están dedicados a la vida de campo, su población considera su comunidad y estilo vida como estable y placentero.

Este pago por el ingreso de visitantes a la EBTC, contribuye a la comunidad San José de Payamino con un promedio anual que va desde los US \$ 3474 a los 5000 en los últimos ocho años. Este rubro es importante, especialmente considerando que este sistema de pago por servicio ambiental puede ser considerado como una propuesta alternativa que puede ser fomentada a nivel comunitario en sitios de importancia biológica y de desarrollo sostenible como la zona de amortiguamiento y transición de las Reservas de Biosfera.

El involucramiento de los miembros de la comunidad en actividades en la EBTC como: guianza, canoeros, actividades de exploración, colocación de cámaras trampa, monitoreo de flora y fauna, es interesante de seguir evaluando con mayor profundidad, dado a que se percibe una atmosfera de conciencia ambiental, que se refleja en la disminución de la tala, caza y pesca que era su principal fuente de ingreso económico anteriormente, con lo cual se crea un valor agregado como comunidad.

La decisión sobre las inversiones de los recursos económicos se toma en las asambleas, y la administración se encarga a la directiva de la comunidad, la cual administra los recursos de manera homogénea beneficiando a toda la población local, donde los principales recursos son destinados a la agricultura, educación y trámites administrativos como comunidad, provocando como resultado una comunidad mejor organizada, que trata de beneficiar a todos sus miembros.

La percepción de la red de actores académicos involucrados califica como "alto" el respeto a los elementos de la cosmovisión indígena en las actividades del TCi realizados en la EBTC. Los ingresos económicos que genera también la existencia de la EBTC beneficia a la comunidad en diversas maneras aun en necesidades secundarias de la población como la agricultura, artículos escolares, salud, etc.

Los habitantes de la comunidad Kichwa San José de Payamino perciben que el funcionamiento de la EBTC contribuye al desarrollo sostenible, científico y económico de la comunidad, creando conciencia en el manejo de los Recursos Naturales vinculando de alguna manera actividades de las cuatro formas del TCi resaltando las costumbres locales comunitarias.

\section{Reconocimientos}

T os autores de este documento expresamos nuestro agradecimiento a la administración de la Estación Biológica Timburi Cocha y a las autoridades de la Universidad Estatal Amazónica por la aprobación del proyecto de Investigación: Condiciones de vida, uso de suelo y turismo científico en la comunidad kichwa San José de Payamino, Amazonía Ecuatoriana. También expresamos nuestro agradecimiento a todos los pobladores de la comunidad kichwa San José de Payamino, así como también a los colegas e investigadores de la UEA: Héctor Reyes y Yasiel Arteaga.

\section{Bibliografía}

Amstrong, R. (2012). An analysis of the conditions for success of community based tourism enterprises. ICRT Occasional Paper, 1-52p.

Bourlon, F., Mao, P., y Quezada, F. (2013). Generando un proceso de Certificación para el Turismo Científico. Explorando las Relaciones entre el Turismo y Ciencia.

Bourlon, F., y Mao, P. (2011). Formas de turismo científico en Aysén, Chile. Nature tourism developmente territorial dynamics of bordelands, 26.

Bursztyn, I., Bartholo, R., \& Gruber, D., (2009). Turismo de base comunitaria diversidad de hogares y experiencias brasileras. Ministerio de Turismo Brasil.

Garcia Mercedes y Martinez Olga. (2017). Turismo Científico y ciudades del Futuro. Internacional Journal of Scientific Management and Tourism. Vol. $3 \mathrm{~N}^{\circ} 1$ pp 123-130

Laarman, J., y Perdue, R. (1989). Science Tourism in Costa Rica. Annals of Tourism Research, 16(2), 205-215. doi:https://doi.org/10.1016/0160-7383 (89)90068-6

López, P. (2013). Ecuador, un laboratorio natural. E-Ciencia Revista de Divulgación Científica de la Escuela Politécnica del Ejército ESPE, (10), 6-13. Recuperado de http:/ugi.espe.edu.ec/ugi/wp-content/uploads/2013/08/ REVISTA-E-CIENCIA-10.pdf

Quesada, R. (2010). Elementos de Turismo: Teoría, Clasificación y Actividad. Editorial. Universidad Estatal a Distancia. Costa Rica.

Pena, M. (2012). Estudio sobre turismo rural sostenible y su aplicación docente (Tesis doctoral). Universidad Almería, España

Revelo, R. (2017). Estudio de los beneficios de tener una Estación Científica en la Amazonía. Revista Publicando, 4(10), 505-519. Recuperado de http://rmlconsultores. com/revista/index.php/crv/article/view/455/pdf 288

Ródas, M., Sanmartín, I., \& Ullauri Donoso, N. (2015). Turismo comunitario en el Ecuador. Una revisión de la literatura. RICIT, 60-77.

Rosyidie, A., Leksono, R., y Adriani, Y. (2012). Scientific 
Tourism Potential in Bandung City. ASEAN Journal on Hospitality and Tourism, 11(2), 129-149. Recuperado de http://journals.itb.ac.id/index.php/ajht/article/view/3469

Ruiz, E., \& Solis, D. (2016). El turismo comunitario en Ecuador. Desarrollo y sostenibilidad social.

Silva, P., (2018). Patrimonio natural y cultural del CIPCA para el desarrollo del turismo científico, como referente en el contexto universitario Amazónico. Proyecto de tesis de maestría. Universidad Estatal Amazónica, Puyo-Ecuador

Torres, B., Gunter, S., Acevedo, R., y Knoke, T. (2018). Livelhood strategies, ethnicity and rural income: The case of migrant settlers and indigenous populations in the Ecuadorian Amazon. Forest Policy and Economics, 22-34.

Vasco, C., Torres, B., Pacheco, P., y Griess, V. (2017). The socioeconomic determinants of legal and ilegal smallholder logging: Evidence from the Ecuadorian Amazon. Forest Policy and Economics, 133-140.

Vasco, C., Bilsborrow, R., y Torres, B. (2015). Income diversificación of migrant colonists vs. indigenous populations: Contrasting strategies in the Amazon. Journal of Rural Studies, 1-10. 